\title{
THE APPLICATION OF SATURATING DETECTORS TO A DCT-DOMAIN WATERMARKING SCHEME
}

\author{
FABING DUAN \\ Institute of Complexity Science, Qingdao University, \\ Qingdao 266071, PR China \\ fabing1974@yahoo.com.cn \\ DEREK ABBOTT \\ Centre for Biomedical Engineering (CBME) and School of Electrical \&S Electronic Engineering, \\ The University of Adelaide, SA 5005, Australia \\ dabbott@eleceng.adelaide.edu.au \\ FRANÇOIS CHAPEAU-BLONDEAU \\ Laboratoire d'Ingénierie des Systèmes Automatisés (LISA), \\ Université d'Angers, 62 avenue Notre Dame du Lac, 49000 Angers, France \\ chapeau@univ-angers.fr
}

Received 10 January 2008

Revised 21 January 2008

Accepted 28 January 2008

Communicated by Igor Goychuk

The present paper proposes a discrete cosine transform (DCT) domain watermarking scheme by exploiting nonlinear dynamical saturating detectors in the design of a watermark detection process. A binary copyright character, i.e. watermark to be hidden into an image, is firstly reordered into a binary zig-zag sequence, and then mapped onto the pulse amplitude modulated signal. A certain desynchronization time delay can be deliberately placed into one code of the modulated signal, and is tolerated due to the superior robustness of nonlinear detectors over matched filters. A selected set of DCT coefficients of a host image in a mid frequency range is shuffled by the Arnold transform, which makes it look more like background noise with respect to the watermark signal. Then, the watermark signal is embedded in the set of shuffled DCT coefficients. The copyright character can be extracted by a nonlinear saturating detector without prior knowledge of the original image and watermark, i.e. blind watermark detection. Interestingly, a higher match between the original watermark character and the extracted one can be further achieved by a parallel array of nonlinear detectors via the mechanism of array stochastic resonance. Robustness of the proposed watermarking scheme is shown in the presence of noise, filtering, cropping, and compression.

Keywords: Saturating detector; watermark detection; Arnold transform; array stochastic resonance. 


\section{Introduction}

Digital watermarking has recently received great attention as a tool for copyright protection [1-10]. A digital watermark contains information about the copyright owner, the authorized consumer or other information that is desired to be embedded. There are two main parts to building a strong watermark: the watermark structure and the insertion strategy [1]. The strong watermark should be robust to various stages of post processing, e.g. compression. The insertion strategy should elicit a convenient watermark detection procedure.

So far, there are two main proposed watermarking techniques, i.e. embedding the watermark in the spatial domain and in the transformed domain of a host image [1-10]. Currently, the later method is generally preferred, e.g. embedding the watermark in the discrete cosine transform (DCT) image domain. There are also two main watermark detection techniques according to the way the watermark is recovered from the possibly distorted version of the marked image, i.e. nonblind and blind watermark detection [1-3]. The approach of extracting the embedded watermark, without comparing the original and the marked images, will be referred to as the blind watermarking detection technique. Moreover, the original watermark is also not needed $[7,8]$.

Digital watermarking systems can be viewed as digital communication systems [6]. Here, the watermark can be regarded as input information, while the DCT coefficients of an image can be thought of as background noise. This viewpoint inspired the application of parameter-induced stochastic resonance [11] to a digital watermark scheme $[9,10]$. The key is how to randomize the DCT coefficients as the background noise, and another factor is the design of the bistable system parameters for detecting the watermark robustly $[9,10]$. In our opinion, there are two main disputes: (i) The nonlinear system is monostable with the selected parameters for a given watermark strength in $[9,10]$. In this case, background noise, i.e. the randomized DCT coefficients, will not play a positive role in the watermark detection. In other words, no conventional stochastic resonance effects occur in the sense of exploiting noise [12]; (ii) This kind of monostable system is more like a nonlinear filter that is suboptimal to a matched filter $[13,14]$. This fact indicates that the design of nonlinear system seems to be unnecessary for the watermark scheme. However, the robustness of the nonlinear system is not considered in [9,10]. These nonlinear systems exhibiting stochastic resonance effects are potentially useful for signal detection, though generally more difficult to theoretically tackle [13-18].

In this paper, we design a blind DCT-domain watermarking scheme integrated with a nonlinear dynamical saturating detector. The efficient signal-processing ability of this kind of dynamical saturating detector proves better than a bistable system in terms of the statistical measure of signal-to-noise ratio [15]. Here, the robustness of this nonlinear saturating detector is extensively considered in the context of a digital watermarking scheme. The watermark consists of a binary copyright character, and is reordered into a zig-zag scan sequence. This sequence is then mapped onto an one-dimensional pulse amplitude modulated (PAM) signal. Moreover, this watermark signal is deliberately desynchronized by inserting a delay time at one arbitrary code, and this saturating detector is demonstrated robust to this degraded condition of desynchronization. From the full-frame DCT domain of an original 
image, we select a set of DCT coefficients from the mid range of the frequency spectrum. These selected DCT coefficients undergo the Arnold transform, and behave more like background noise. Then, the watermark signal is superimposed on the disordered DCT coefficients, resulting in the marked DCT coefficients. In succession, the marked DCT coefficients return to their corresponding positions via the inverse Arnold transform, and replace the selected set of DCT coefficients of the original image. After the inverse transform of the marked full-frame DCT coefficients, the marked image is obtained. The proposed watermarking scheme is tested on some common forms of interference and distortion: additive Gaussian noise, histogram equalization, JPEG compression, low pass and high pass filtering, geometric cropping, salt \& pepper noise, multiplicative speckle noise, and multiple watermarking. Given various corrupted marked images, the watermark can be recovered by reversing the watermark embedding procedure. First, the corrupted DCT coefficients embedded with the watermark can be obtained. Then, it is applied to an isolated saturating detector or a parallel array of nonlinear saturating detectors for extracting the watermark, i.e. the copyright character. Finally, this watermark scheme is evaluated by measuring the similarity between the extracted character and the original one. Due to the special watermark structure, this method makes the nonlinear detector especially suited to the problem compared to a matched filter. Interestingly, we observe that array stochastic resonance $[17,18]$, by a parallel array of nonlinear saturating detectors, can further improve the similarity measure to a higher level than an isolated detector does. It is emphasized that the internal array noise plays a constructive role in the watermark detection and acts as an operational tool for improving the performance of the detectors. This application of saturating systems to DCT domain watermarking detection indicates that nonlinear systems are potential signal processors, and the extended stochastic resonance is a useful mechanism that deserves to be further studied in signal processing field.

\section{Watermark Embedding}

\subsection{Structure of watermark}

Generally, a pseudo-random binary number is used as the watermark $[1,2]$. Here, a black-and-white copyright character $S$ of size $M \times M$, as shown in Fig. 1 (a), is employed as a binary watermark. Since 0 s and 1 s correspond to black and white pixels, or conversely, this copyright character $S$ contains $M^{2}$ bits. This two-dimensional binary image $S$ is firstly reordered into an one-dimensional zig-zag sequence, such as in the JPEG compression algorithm [2]. Next, we modulate this binary zig-zag sequence onto a PAM signal with amplitude $\pm A$ and bit interval $T_{b}$. Ten examples of the PAM waveforms are shown in Fig. 1 (b). Each waveform lasts for a bit interval $T_{b}$, but an arbitrary waveform is deliberately delayed by $0.5 T_{b}$, as shown in Fig. 1 (b). This kind of desynchronized PAM signal is specially designed for the watermarking detection scheme. Experimental results also demonstrate in Sect.4 that this desynchronized PAM signal has little effect on the detection of a nonlinear saturating detector. In succession, the PAM waveform is sampled with a sampling time $0.01 T_{b}$. Thus, each pulse contains 100 sample values and a sample signal vector $X=\left\{x_{1}, x_{2}, \cdots, x_{100 M^{2}}\right\}$ is obtained. Note that the last PAM waveform is a half bit interval having 50 sampled points. In this way, a copyright character $S$ will be 
(a)

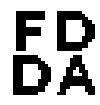

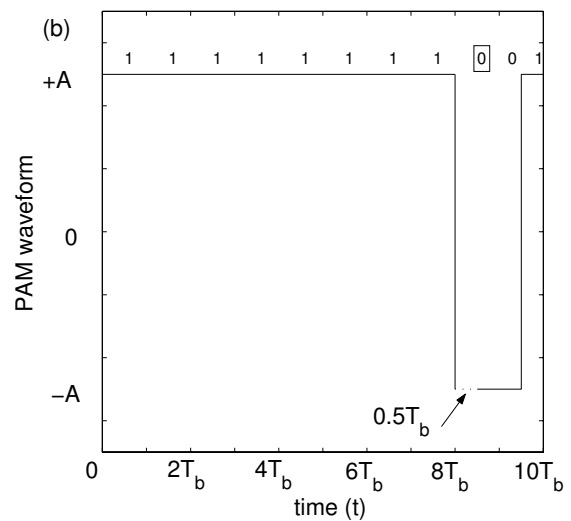

Fig. 1. (a) Binary copyright character $S$ with size of $29 \times 29(M=29)$. (b) Example of PAM waveform encoded by the zig-zag version of the copyright character. Here, the pulse amplitude is $\pm A$ and the bit interval is $T_{b}$. One code of bit 0 is deliberately desynchronized by a delay time of $0.5 T_{b}$, as indicated by the arrow.

(a)

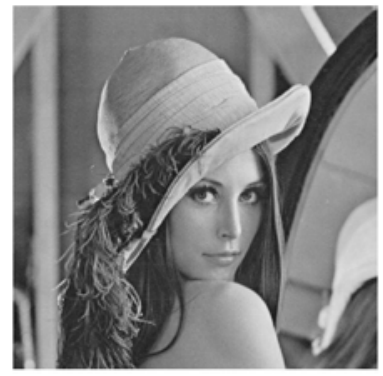

(b)

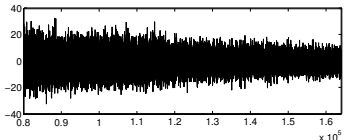

(c)

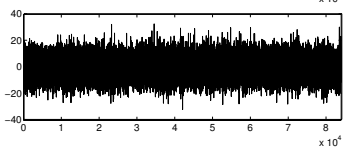

(d)

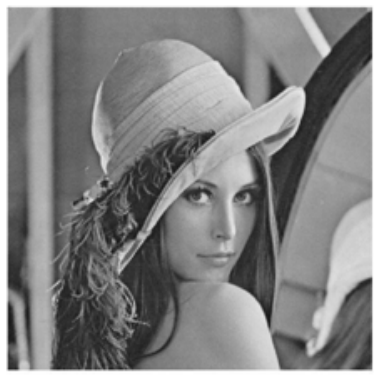

Fig. 2. (a) Host image 'Lena' $I$ with size of $512 \times 512(N=512)$. (b) Selected set of zig-zag scan of DCT coefficients as a function of the index. (c) The shuffled DCT coefficients sequence of $T$ after Arnold transform. (d) Marked image $I^{\prime}$. Here, the PAM signal has amplitude $A=4$ with the same unit of DCT coefficients.

transformed into a watermark vector $X$ to be embedded into the DCT domain of an image.

\subsection{Watermark casting}

In this step, the $N \times N$ DCT coefficients for a $N \times N$ gray-scale image $I$, as shown in Fig. 2 (a), are computed. The definition of the two-dimensional DCT for an input image $I(i, j)$ and output coefficients $G(m, n)$ is

$$
\begin{aligned}
G(m, n) & =\alpha(m) \alpha(n) \sum_{i=0}^{N-1} \sum_{j=0}^{N-1} I(i, j) \cos \left[\frac{\pi(2 i+1) m}{2 N}\right] \cos \left[\frac{\pi(2 j+1) n}{2 N}\right], \\
\alpha(0) & =\sqrt{1 / N}, \\
\alpha(m) & =\sqrt{2 / N}, \quad 1 \leq m \leq N,
\end{aligned}
$$


where $m$ and $n$ are the row and column indices of $G$, respectively. Similarly, $i$ and $j$ are the row and column indices of $I$. The DCT coefficients are also reordered into a zig-zag sequence, and the first DCT coefficient, i.e. the DC term, should be not modified [1-8]. The coefficients from the $(L+1)$ th to the $\left(L+100 M^{2}\right)$ th positions are taken out according to the above zig-zag sequence of the DCT spectrum, as shown in Fig. 2 (b). There are $100 M^{2}$ DCT coefficients in total to be modified.

In this paper, when considering stochastic resonance, we take the binary information as the input signal, and view the selected DCT coefficients as noise. However, as shown in Fig. 2 (b), the selected DCT coefficients are not sufficiently like white noise. In order to address this problem, we reshape the selected DCT coefficients into a $10 M \times 10 M$ matrix. Then, this reordered matrix is shuffled by the Arnold transform [8]

$$
\left[\begin{array}{l}
x^{\prime} \\
y^{\prime}
\end{array}\right]=\left[\begin{array}{ll}
1 & 1 \\
1 & 2
\end{array}\right]\left[\begin{array}{l}
x \\
y
\end{array}\right](\bmod 10 M),
$$

where $x, y \in[1,2, \cdots, 10 M]$ are the pixel coordinates of the reshaped matrix, and $x^{\prime}, y^{\prime} \in[1,2, \cdots, 10 M]$ correspond to the shuffled DCT coefficient matrix. Here, the Arnold transform iterated $k$ times. Note that $p=k+m$ Arnold transforms for a $10 M \times 10 M$ matrix of DCT coefficients [8] will return to their original positions.

Next, the $10 M \times 10 M$ shuffled matrix of DCT coefficients is reordered into a zig-zag sequence $T=\left\{t_{1}, t_{2}, \cdots, t_{100 M^{2}}\right\}$, as plotted in Fig. 2 (c). $T$ is more like white noise than the original zig-zag scan of Fig. 2 (b). Then, the input signal vector $X$ is added to the zig-zag sequence of $T$. Thus, the marked DCT coefficient sequence $T^{\prime}$ is given as

$$
T^{\prime}=T+X
$$

Finally, the modified sequence $T^{\prime}=\left\{t_{1}^{\prime}, t_{2}^{\prime}, \cdots, t_{100 M^{2}}^{\prime}\right\}$ is reinserted in the inverse zig-zag scan. Then, the $10 M \times 10 M$ marked matrix of DCT coefficients are embedded, and undertake the residual $m$ Arnold transform iterations. We replace the $10 M \times 10 M$ embedded DCT coefficients with the original ones in the full-frame DCT domain, and perform the inverse $N \times N$ DCT transform. Then, the marked image is obtained. For example, a wartermarked image $I^{\prime}$ is shown in Fig. 2 (d). The peak signal-to-noise (PSNR) of this marked image $I^{\prime}$ is $40.75 \mathrm{~dB}$ [1-10].

\section{Watermark Detection and the Measure of Similarity}

The marked image $I^{\prime}$ is possibly corrupted by some signal post processing, e.g. compression, and becomes a corrupted image $I^{*}$. The $N \times N$ DCT transform is applied to the marked image $I^{*}$. Then, the corrupted $N \times N$ DCT coefficients embedded with watermarks can be obtained by reversing the steps of watermark casting in Sec. 2. It is reordered into a zig-zag sequence and coefficients from the $(L+1)$ th to the $\left(L+100 M^{2}\right)$ th positions are selected. These $100 M^{2}$ coefficients are reshaped into a $10 M \times 10 M$ matrix via the inverse of the zig-zag procedure, and the formed matrix undergoes $k$ iterations of the Arnold transform. Then, this shuffled matrix is reordered into the zig-zag sequence $T^{*}=\left\{t_{1}^{*}, t_{2}^{*}, \cdots, t_{100 M^{2}}^{*}\right\}$ to be detected for revealing the watermark. In order to extract the recovered binary watermarked image $S^{*}$, we utilize two alternative detection strategies as follows. 


\subsection{Isolated nonlinear dynamical saturating detector}

In this detection task, we apply $T^{*}$ to a nonlinear dynamical saturating detector given as [15]

$$
\tau_{a} \frac{d x(t)}{d t}=-x(t)+\left[1-\frac{x^{2}(t)}{X_{b}^{2}}\right] T^{*}(t),
$$

with detector parameters $\tau_{a}>0$ and $X_{b}>0$. Equation (4) exhibits saturating dynamics: When $|x(t)| \ll X_{b}$, then Eq. (4) reduces to linear dynamics where $\tau_{a} d x(t) / d t \approx-x(t)+T^{*}(t)$, by which $x(t)$ tends to follow the noisy input $T^{*}(t)$ within the lag imposed by the time constant $\tau_{a}$; when $x(t)$ approaches $\pm X_{b}$, then the term $x(t)^{2} / X_{b}^{2}$ is close to one, the factor $\left[1-x(t)^{2} / X_{b}^{2}\right]$ is close to zero and tends to reduce and turn off the action of the noisy input [15]. This is the saturation effect. Strictly, when $x(t)$ reaches $\pm X_{b}$, the action of the noisy input is turned off, and $x(t)$ starts to relax to zero. By this mechanism, the dynamics of Eq. (4) when initialized at $x(0) \in]-X_{b}, X_{b}$ [ can never exceed $\pm X_{b}$, and the time evolution of $x(t)$ remains confined to $\left[-X_{b}, X_{b}\right]$. Thus, the dynamics of Eq. (4) is linear at small $x(t)$ and saturates when $x(t)$ approaches $\pm X_{b}$ [15]. This is the dynamic analog of the static saturating nonlinearity of [16]. Here, the differential equation of Eq. (4) is written in continuous form, and the vector $T^{*}$ is represented as a continuous input signal $T^{*}(t)$. In this paper, we numerically integrate Eq. (4) using Euler-Maruyama discretization with a sampling time step $\Delta t \ll T_{b}[13-15,17,18]$. Throughout the paper, we adopt $\Delta t=0.01 T_{b}$. The output $x(t)$ is sampled for recovery of the watermark.

\subsection{An uncoupled parallel array of saturating detectors}

In our previous work in the area of array stochastic resonance [18], we have reported that signal-to-noise ratio gain exceeding unity is achievable when a parallel array of nonlinear dynamical subsystems is subjected to a noisy subthreshold or suprathreshold input. Here, an uncoupled parallel array of saturating detectors is considered for obtaining an improved binary mark image versus an isolated detector of Eq. (4). Zero-mean Gaussian white noise $\eta_{i}(t)$, together with and independent of $T^{*}(t)$, is applied to each detector of the parallel array of size $K$. The $K$ array noise terms $\eta_{i}(t)$ are mutually independent and have autocorrelation $\left\langle\eta_{i}(t) \eta_{i}(0)\right\rangle=D_{\eta} \delta(t)$ with a same noise intensity $D_{\eta}$. The internal state $x_{i}(t)$ of each saturating detector is governed by

$$
\tau_{a} \frac{d x_{i}(t)}{d t}=-x_{i}(t)+\left[1-\frac{x_{i}^{2}(t)}{X_{b}^{2}}\right]\left[T^{*}(t)+\eta_{i}(t)\right],
$$

for $i=1,2, \ldots, K$. Their outputs are averaged and the response of the array is given as

$$
y(t)=\frac{1}{K} \sum_{i=1}^{K} x_{i}(t) .
$$

Then, the response $y(t)$ of Eq. (6) is subsequently sampled in order to recover the watermark. 


\subsection{Recovering procedure and the similarity measure}

We introduce the detection scheme as follows $[13,14,17,18]$ : the output $x(t)$ of Eq. (4) or the average output $y(t)$ of Eq. (6) is sampled at $j T_{b}$ for $j=1,2, \cdots, M^{2}$, resulting in a successive sampled values of $Y_{j}$. Note that the sample points take on the corresponding numerical values at $j T_{b}$, regardless of the desynchronization time. This sampling method has a weak effect on the saturating detector, but a deleterious effect on a matched filter $[13,14]$. Thus, the saturating detector is utilized as a novel robust detector in this watermark scheme. Next, the $Y_{j}$ are compared with the decision threshold $\ell$ for selecting binary values 0 or 1 . If $Y_{j}>\ell$, the recovered mark is read as a digital 1 , otherwise a digital 0 . Here, we utilize the hard thresholding method of $\ell=0$ [14]. Now, this system of Eq. (4) or Eq. (5) with input binary signal and output reading binary bits, can be viewed as an information channel transmitting binary data.

Following the above detection scheme, we obtain a recovered binary sequence. This binary sequence is then reshaped by the inverse zig-zag scan, and the recovered binary image of copyright character $S^{*}$ can be formed. The similarity $\rho$ between the original character $S$ and the recovered one $S^{*}$ is measured by means of the formula

$$
\rho=\frac{S \cdot S^{*}}{S^{*} \cdot S^{*}}=\frac{\sum \sum s s^{*}}{\sum \sum s^{* 2}},
$$

where $s$ and $s^{*}$ are the pixel values of $S$ and $S^{*}$, respectively. We compare $\rho$ with a threshold to decide how similar the recovered mark $S^{*}$ is to the original $S$. The threshold has a range from zero to unity, and the exact value of the threshold depends on the requirements. The larger $\rho$ is, the more similar $S^{*}$ is to $S$.

\section{Experimental Results}

In order to evaluate the proposed watermarking scheme, a $29 \times 29$ copyright character $S$ was embedded into the $512 \times 512$ grey-scale standard image 'Lena' $I$, as shown in Figs. 1 and 2. Several common signal processing techniques and geometric distortions are applied to the marked image to evaluate if the nonlinear saturating detector of Eq. (4) or Eq. (5) can reveal the embedded mark well or not. The similarity measure will enable the robustness of this watermarking scheme to be evaluated.

In this paper, main experimental results using the standard image 'Lena' are presented. The saturating detector has tunable parameters $\tau_{a}$ and $X_{b}$ [15]. The $\tau_{a}$ and $X_{b}$ parameter tuning method is described by Duan et al [14]. The noise intensity $D_{\eta}$ is also a tunable parameter for the array of saturating detectors $[17,18]$.

The JPEG compression algorithm is one of the most severe forms of distortion that the watermark meets. We applied JPEG coding with decreasing quality and $0 \%$ smoothing to the marked image $I^{\prime}$. Here, the JPEG compression quality means unity minus the compression ratio expressed in percentage. An image compression example with $65 \%$ quality and $0 \%$ smoothing is shown in Fig. 3 (a). The PSNR is degraded as $36.31 \mathrm{~dB}$. The isolated saturating detector detects the binary mark image with similarity $\rho=0.7950$, while the array of nonlinear detectors of Eq. (6) 
(a)

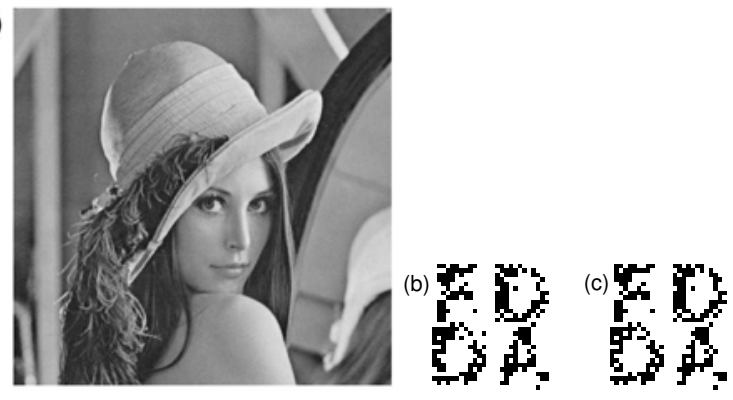

Fig. 3. (a) JPEG compressed version of the marked image 'Lena' with $65 \%$ quality and $0 \%$ smoothing. (b) The recovered binary image via the isolated saturating detector, and (c) the array of saturating detectors at the optimal noise intensity $D_{\eta} / \Delta t=5$. Here, $\tau_{a}=0.05 T_{b}, X_{b}=0.25 A$ and the array size $K=60$.

(a)

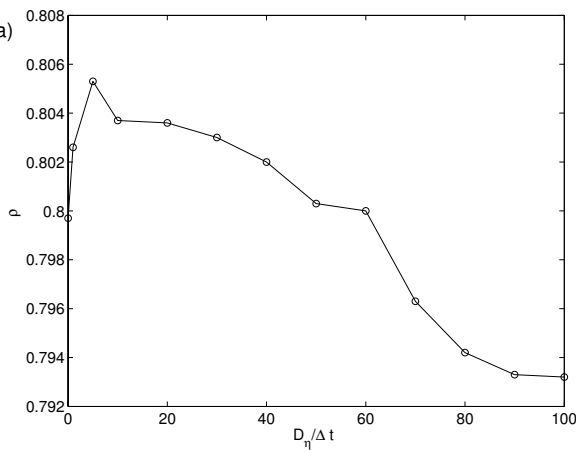

(b)

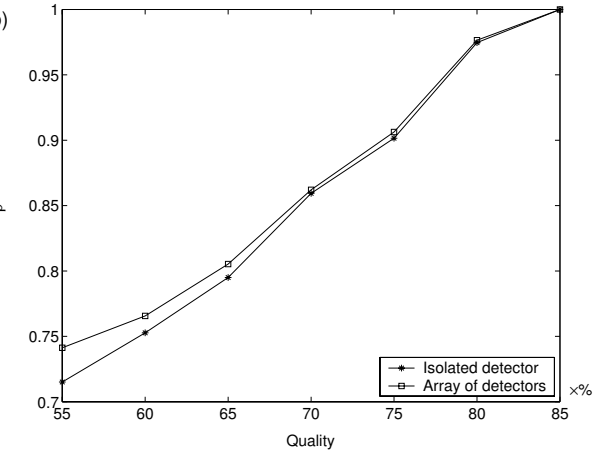

Fig. 4. (a) Similarity $\rho$ as a function of array noise density $D_{\eta} / \Delta t$. The JPEG compression quality is $65 \%$. (b) Similarity $\rho$ as a function of JPEG quality. Each point of $\rho$ (squares) obtained by array of saturating detectors is observed indicated in the way of Fig. 4 (a). Other parameters are same as in Fig. 3.

tells $\rho=0.8053$ at an optimal noise intensity $D_{\eta} / \Delta t=5$. Both recovered watermark images $S^{*}$ are shown in Fig. 3 (b) and (c), respectively. By comparing each pixel of $S^{*}$ with $S$, Fig. 3 (c) has more than eight correct pixels than Fig. 3 (b) does.

Note that $D_{\eta}$ is an independent tunable parameter and is determined in the following way: Upon increasing $D_{\eta}$ from zero, as shown in Fig. 4 (a), $\rho$ shows a resonance-like curve; We select the highest value of similarity $\rho=0.8053$, and then obtain the corresponding optimal value of $D_{\eta} / \Delta t=5$. Furthermore, the measure of $\rho$ is plotted in Fig. 4 (b) as the monotonic increasing function of JPEG compression quality. It is obvious that the array of saturating detectors presents a better result than an isolated saturating detector does. The JPEG compression algorithm usually discards the high-frequency DCT coefficients. The addition of array noise, as indicated in Fig. 4 (b), can help the nonlinear detectors recover the watermark signal. This mechanism is referred to array stochastic resonance [18]. 


\subsection{JPEG compression}

\subsection{Histogram equalization}

As shown in Fig. 5 (a), the marked image $I^{\prime}$ is operated by histogram equalization into $I^{*}$ with the PSNR being $19.04 \mathrm{~dB}$. The isolated saturating detector presents the estimate watermark $S^{*}$, as shown in Fig. $5(\mathrm{~b})$, with the similarity $\rho=1.0$. Thus, it is no necessary to adopt an array of nonlinear detectors of Eq. (6) for the watermark detection.

(a)

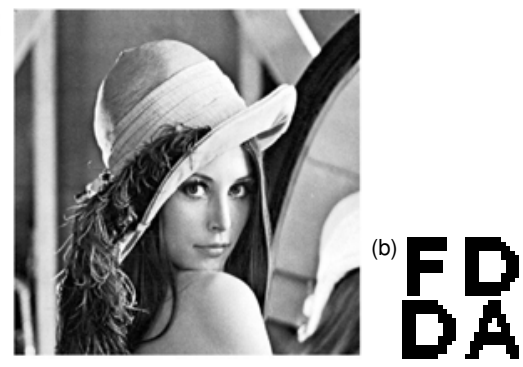

Fig. 5. (a) Watermarked image 'Lena' after histogram equalization. (b) The recovered binary image detecting by the isolated saturating detector. Here, $\tau_{a}=0.05 T_{b}$ and $X_{b}=0.25 A$.

\subsection{Adding Gaussian noise}

Zero-mean Gaussian noise with variance $\sigma^{2}$ is introduced to corrupt the marked image $I^{\prime}$ in the spatial domain. As the variance $\sigma^{2}$ increases, the image degradation is more severe. An example corrupted image $I^{*}$ is illustrated in Fig. 6 (a) for Gaussian noise variance $\sigma^{2}=225$. The PSNR of the corrupted image $I^{*}$ is $24.47 \mathrm{~dB}$. The isolated saturating detector detects the binary mark image with similarity $\rho=0.9839$, as shown in Fig. 6 (b). While the array of saturating detectors of Eq. (6) gives $\rho=0.9890$ at an optimal noise intensity $D_{\eta} / \Delta t=100$, as shown in Fig. 6 (c). By comparing the recovered watermark $S^{*}$ of Fig. 6 (b) and (c) with $S$, it is observed that Fig. 6 (c) can correct three erroneous pixels in Fig. 6 (b).

As the marked image $I^{\prime}$ is corrupted at a different Gaussian noise variance $\sigma^{2}$, we follow the way of tuning noise intensity $D_{\eta} / \Delta t$ indicated in Fig. 4 (a). The corresponding optimal similarity $\rho$ is plotted in Fig. 6 (d) for the array of saturting detectors (squares). For comparison, the isolated saturting detector (stars) is also drawn in Fig. $6(\mathrm{~d})$. It is seen that the array noise $\eta_{i}(t)$ slightly improves the similarity $\rho$ via the mechanism of array stochastic resonance [18] at low Gaussian noise intensity. Besides, experimental results show that, even at $\sigma^{2}=2600$, the nonlinear detector is able to recover the character with the similarity $\rho=0.8022$, as shown in Fig. 6 (d).

\subsection{Geometric cropping}

We cropped the marked image $I^{\prime}$ as a $256 \times 256$ subimage $I^{*}$, i.e. $25 \%$ of $I^{\prime}$, as shown in Fig. 7 (a). The PSNR is $6.56 \mathrm{~dB}$. The corresponding recovered watermarks $S^{*}$ are illustrated in Fig. 7 (b) and (c), with similarity $\rho=0.9124$ and $\rho=0.9408$, respectively. It is observed that the addition of array noise is obviously helpful 
(a)

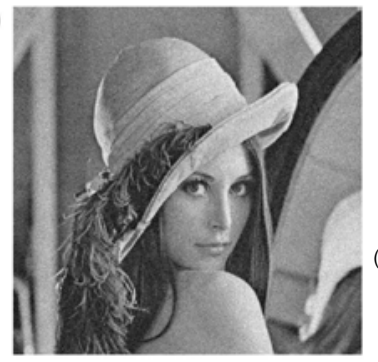

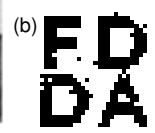
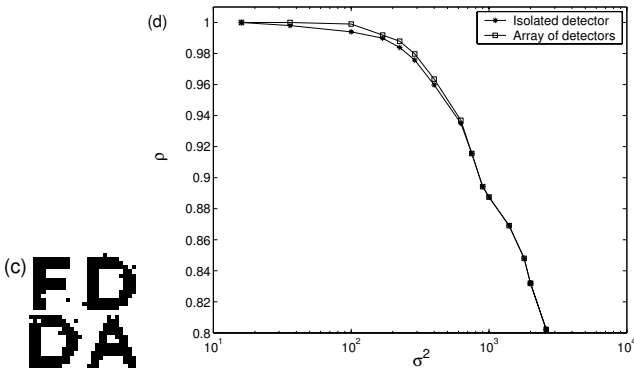

Fig. 6. (a) Watermarked image 'Lena' corrupted by Gaussian noise with variance $\sigma^{2}=225$. (b) The recovered binary image via the isolated saturating detector, and (c) by the array of saturating detectors at the optimal noise intensity $D_{\eta} / \Delta t=100$. (d) Similarity $\rho$ as a function of Gaussian noise variance $\sigma^{2}$. Here, $\tau_{a}=0.1 T_{b}, X_{b}=0.25 A$ and the array size $K=60$.

(a)
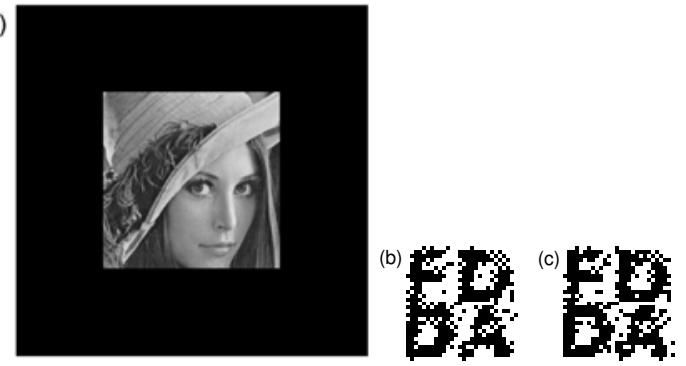

Fig. 7. (a) The marked image 'Lena' after cropping as a $256 \times 256$ subimage. The black background indicates the cutting section. (b) The recovered binary image $S^{*}$ via the isolated saturating detector and (c) the array of saturating detectors at the optimal noise intensity $D_{\eta} / \Delta t=300$. Here, $\tau_{a}=0.1 T_{b}, X_{b}=0.25 A$ and the array size $K=60$.

to the detectability of nonlinear detectors. As a consequence, the scheme behind Fig. 7 (c) has corrected over ten erroneous pixels compared to that in Fig. 7 (b).

\subsection{Low pass filtering and high pass filtering}

The marked image $I^{\prime}$ is filtered by a low pass filter with window size $3 \times 3$, as shown in Fig. 8 (a). The PSNR is $31.87 \mathrm{~dB}$. The corresponding recovered watermarks $S^{*}$ are also illustrated in Fig. 8 (b) and (c), with similarity $\rho=0.8528$ and $\rho=0.8839$, respectively. Figure. 8 (b) corrects twenty five erroneous pixels in Fig. 8 (c) with the help of the array noise. This watermarking scheme fails under the low pass filter with window size $5 \times 5$.

The marked image $I^{\prime}$ is also filtered by a high pass filter with window size $4 \times 4$, as shown in Fig. 9 (a). The PSNR is $30.51 \mathrm{~dB}$. The corresponding recovered watermark $S^{*}$ is illustrated in Fig. 9 (b) via an isolated detector with similarity $\rho=1.0$. Note that this watermarking scheme failed under the median filter. The main reason is the mark $S$ is embedded in median frequency DCT domain. 
(a)

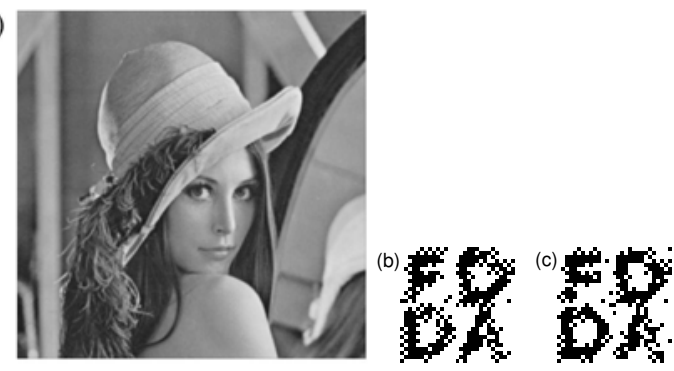

Fig. 8. (a) The marked image 'Lena' filtered by a low pass filter with window size $3 \times 3$. (b) The recovered binary image $S^{*}$ via the isolated saturating detector, and (c) the array of saturating detectors at the optimal noise intensity $D_{\eta} / \Delta t=30$. Here, $\tau_{a}=0.1 T_{b}, X_{b}=0.125 A$ and the array size $K=60$.

(a)

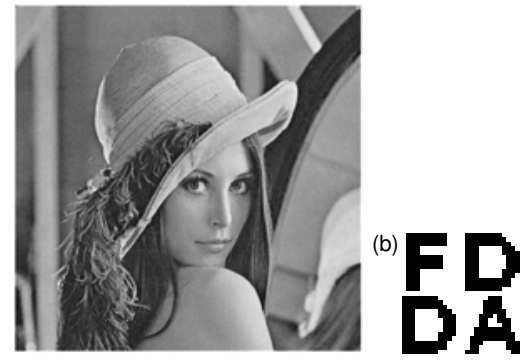

Fig. 9. (a) The marked image 'Lena' filtered by a high pass filter with window size $3 \times 3$. (b) The recovered binary image $S^{*}$ by the isolated saturating detector. Here, $\tau_{a}=0.1 T_{b}$ and $X_{b}=0.125 A$.

\subsection{Salt $\&$ pepper and multiplicative speckle noise}

We corrupt the marked image with salt \& pepper noise and noise density is 0.05 [20,21], as shown in Fig. 10 (a). The PSNR is $18.51 \mathrm{~dB}$. The corresponding recovered watermarks are also illustrated in Fig. 10 (b) and (c), with similarity $\rho=0.8805$ and $\rho=0.9121$, respectively. More than twenty two erroneous pixels in Fig. 10 (b) are corrected in Fig. 10 (c) by the constructive role of array noise.

We corrupt the marked image with multiplicative speckle noise and noise variance is 0.06 [20,22], as shown in Fig. 11 (a). The PSNR is $18.07 \mathrm{~dB}$. The corresponding recovered binary characters are also illustrated in Fig. 11 (b) and (c), with similarity $\rho=0.8792$ and $\rho=8922$. The addition of the array noise helps the eleven erroneous pixels of Fig. 11 (b) be corrected in Fig. 11 (c).

\subsection{Dithering}

Figure 12 (a) shows a dithered version of the 'Lena' image. Here, the marked image $I^{\prime}$ is converted to the binary (black-and-white) image $I^{*}$ by the dithering $[19,20]$. The details of the dithering algorithm, applied to the image, are described by Floyd and Steinberg [19]. The PSNR is $5.69 \mathrm{~dB}$. Once again, the isolated saturating detector tells the recovered character with similarity $\rho=0.8289$, as illustrated in Fig. 12 (b). The recovered character $S^{*}$ is a little obscure, and the constructive role 
(a)

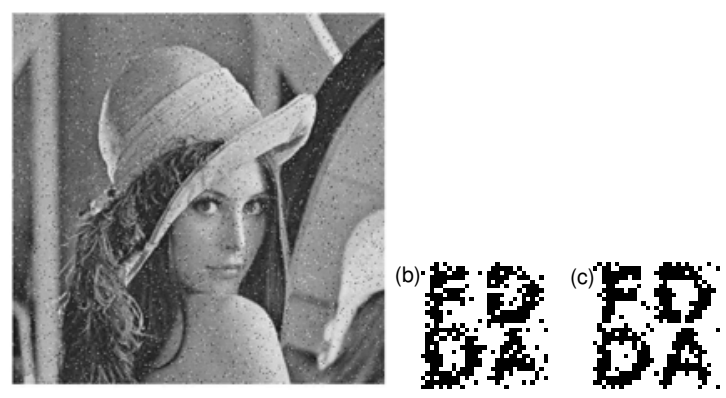

Fig. 10. (a) The marked image 'Lena' added by salt \& pepper noise with noise density 0.05 . (b) The recovered binary image $S^{*}$ via the isolated saturating detector, and (c) the array of saturating detectors at the optimal noise intensity $D_{\eta} / \Delta t=0.5$. Here, $\tau_{a}=0.1 T_{b}, X_{b}=A /(4 \sqrt{2})$ and the array size $K=60$.

(a)

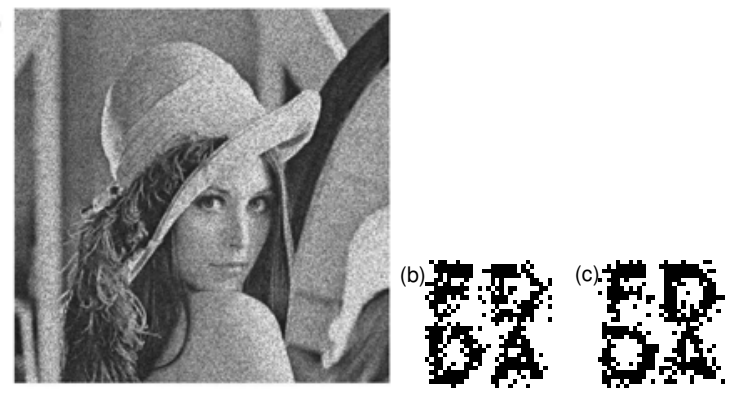

Fig. 11. (a) The marked image corrupted by multiplicative speckle noise with noise variance 0.06. (b) The recovered binary image $S^{*}$ via the isolated saturating detector, and (c) the array of saturating detectors at the optimal noise intensity $D_{\eta} / \Delta t=10$. Here, $\tau_{a}=0.1 T_{b}, X_{b}=A /(4 \sqrt{2})$ and the array size $K=60$.

(a)

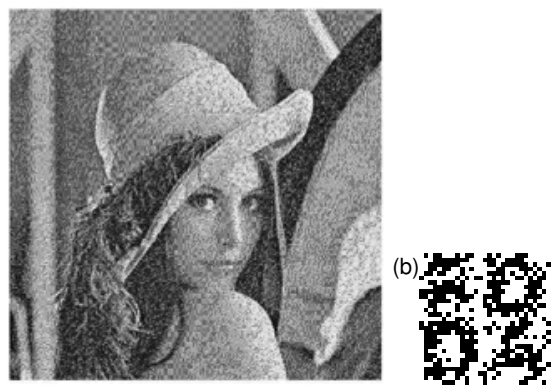

Fig. 12. (a) The marked image after dithering. (b) The recovered binary image by the isolated saturating detector. Here, $\tau_{a}=0.05 T_{b}$ and $X_{b}=A / 4$. 
of array noise fails to give sufficient improvement in this condition. Thus, the array of saturating detectors presents no result.

\subsection{Multiple marks and forgery attacks}

Some applications require that more than one watermark is embedded into a host image $[1,2]$. Besides, one may know the watermark scheme and attempt to insert another watermark aiming at making the original mark unreadable. To test the proposed watermarking scheme under this kind of attack, the marked image $I^{\prime}$ was watermarked again by a random binary sequence with $L=841$ bits as the marked image $I_{1}^{*}$. Then, $I_{1}^{*}$ was watermarked again by another random binary sequence with $L=841$ bits as the marked image $I_{2}^{*}$, and so on until the marked image $I_{5}^{*}$ with five different random binary sequences. $I_{5}^{*}$ is shown in Fig. 13 (a), and its PSNR is $31.92 \mathrm{~dB}$. The watermark cast procedure is as same as that in Subsection 2.2 , but the Arnold transform iteration is the secret key unknown for different watermarking cast procedures. Figure 13 (b) shows the recovered character $S^{*}$ with similarity $\rho=0.9899$ from image $I_{5}^{*}$. The corresponding recovered watermark by array of nonlinear detectors, as illustrated in Fig. 13 (c), has similarity $\rho=0.9960$. The addition of the array noise helps the seven erroneous pixels of Fig. 13 (b) to be corrected in Fig. 13 (c). Moreover, the five test random embedding binary sequence are with similarity values $\rho=0.9222,0.8268,0.9075,0.8190$ and 0.8995 , respectively. If they represent some characters as the original $S$ does, the similarity $\rho$ can be considered sufficient.

(a)

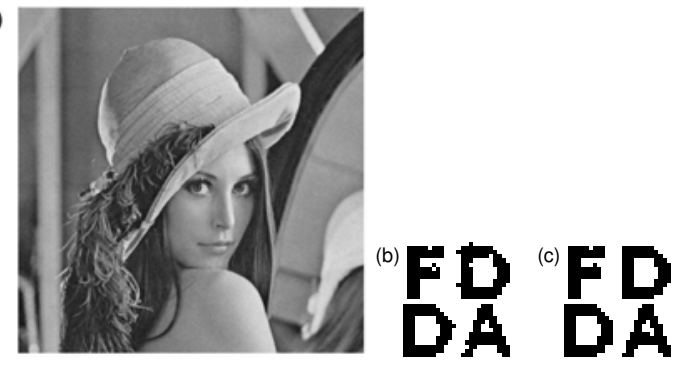

Fig. 13. (a) The marked image embedded with five different watermarks. (b) The recovered binary image $S^{*}$ via the isolated saturating detector, and (c) the array of saturating detectors at the optimal noise intensity $D_{\eta} / \Delta t=1$. Here, $\tau_{a}=0.1 T_{b}, X_{b}=A / 4$ and the array size $K=60$.

\section{Conclusion}

This paper proposed a blind watermark detection scheme for digital gray-scale images operating in the DCT domain: a binary character is embedded in a selected set of DCT coefficients and a nonlinear saturating detector is employed for watermark detection. The character mark is reordered in a zig-zag sequence, and this sequence is then modulated as a PAM signal. This watermark signal is delibertely desynchronized by a delay time at one arbitrary code to test robustness. The fullframe DCT coefficients are also arranged in a zig-zag scan, and extracted in the mid frequency domain. The extracted set of DCT coefficients is further shuffled by the Arnold transform, acting as the background noise for the watermark signal. The watermark signal is then added to the shuffled DCT coefficients. The 
watermark detection employs a saturating detector and a parallel array of saturating detectors since the nonlinear saturating detector is robust to the delay time in the PAM watermark signal. Experimental results demonstrate that this watermark scheme is robust to several signal processing techniques, such as JPEG compression, histogram equalization, additive Gaussian noise, low pass and high pass filtering, geometric cropping, salt \& pepper noise, multiplicative speckle noise, dithering and multiple watermarking. Interestingly, the array of saturating detectors has a tunable parameter of array noise for improving the similarity of the recovered character to the original one. This mechanism is referred to array stochastic resonance.

It is noted that this watermarking algorithm based on saturating detectors needs to be improved to resist attacks of the media filtering and geometric rotating. Research could also find the application of this watermarking algorithm to the color image. Future research of this scheme deserves deeper investigation in embedding the binary information in discrete wavelet transform (DWT) coefficients by employing the dynamical or static saturating detector.

\section{Acknowledgements}

This work is sponsored by NSFC (No. 60602040), Taishan Scholar CPSP, the SRF for ROCS, SEM and PhD PFME of China (No. 20051065002). Funding from the Australian Research Council (ARC) is gratefully acknowledged.

\section{References}

[1] I. J. Cox, J. Kiliant, T. Leighton and T. Shamoon, Secure spread spectrum watermarking for multimedia, IEEE Trans. on Image Processing 6 (1997) 1673-1687.

[2] M. Barni, F. Bartolini, V. Cappellini and A. Piva, A DCT-domain system for robust image watermarking, Signal Processing 66 (1998) 357-372.

[3] M. Barni, F. Bartolini, A.D. Rosa and A. Piva, Capacity of full frame DCT image watermarks, IEEE Trans. on Image Processing 9 (2000) 1450-1455.

[4] E.Y. Lam and J.W. Goodman, A mathematical analysis of the DCT coefficient distributuions for images, IEEE Trans. on Image Processing 9 (2000) 1661-1666.

[5] S. Stanković, I. Djurović, R. Herpers and L. Stanković, An approach to optimal watermark detection, Int. J. Electron. Commun. 57 (2003) 355-357.

[6] S. Baudry, J.F. Delaigle, B. Sankur, B. Macq and H. Maitre, Analyses of error correction strategies for typical communication channels in watermarking, Signal Processing 81 (2001) 1239-1250.

[7] I. Katzenbeisser, S. Petitcolas and A. P. Fabien, Information Hiding Techniques for Steganography and Digital Watermarking (Artech House, Inc, 2000).

[8] M. Barni, F. Bartolini, Watermarking Systems Engineering (Marcel Dekker, Inc, 2004).

[9] S. Sun and P. Qiu, Algorithm of digit watermarking based on parameter-induced stochastic resonance, J. Communications 26 (2005) 48-55.

[10] G. Wu and Z. Qiu, A novel watermarking scheme based on stochastic resonance, IEEE the 8th International Conference on Signal Processing 2 (2006) 16-20.

[11] F. Duan and B. Xu, Parameter-induced stochastic resonance and baseband binary signal transmission over an AWGN channel, Int. J. Bifurcation and Chaos 13 (2003) $411-425$. 
[12] B. Xu, F. Duan and F. Chapeau-Blondeau, Comparison of aperiodic stochastic resonance in a bistable system by adding noise and tuning system parameters, Phys. Rev. E 69 (2004) 061110.

[13] D. Rousseau, J. Rojas Varela, F. Duan and F. Chapeau-Blondeau, Evaluation of a nonlinear bistable filter for binary signal detection, Int. J. Bifurcation and Chaos $\mathbf{1 5}$ (2005) 667-679.

[14] F. Duan, D. Abbott and Q. Gao, Evaluation of bistable systems versus matched filters in detecting bipolar pulse signals, Fluctuation and Noise Lett. 5 (2005) L127-L142.

[15] F. Chapeau-Blondeau, F. Duan and D. Abbott, Signal-to-noise ratio of a dynamical saturating system: Switching from stochastic resonator to signal processor, Physica A 387 (2008) 2394-2402.

[16] F. Chapeau-Blondeau and D. Rousseau, Noise-aided SNR amplification by parallel arrays of sensors with saturation, Phys. Lett. A 351 (2006) 231-237.

[17] F. Chapeau-Blondeau and D. Rousseau, Enhancement by noise in parallel arrays of sensors with power-law characteristics, Phys. Rev. E 70 (2004) 060101(R).

[18] F. Duan, F. Chapeau-Blondeau and D. Abbott, Noise improvement of SNR gain in parallel array of bistable dynamic systems by array stochastic resonance, Electronics Lett. 42 (2006) 1008-1009.

[19] R.W. Floyd and L. Steinberg, An adaptive algorithm for spatial gray scale, International Symposium Digest of Technical Papers 17 (1975) 36-37.

[20] J. S. Lim, Two-Dimensional Signal and Image Processing (Englewood Cliffs, NJ, Prentice Hall, 1990).

[21] R. H. Chan, C. Ho and M. Nikolova, Salt-and-Pepper noise removal by median-type noise detectors and edge-preserving regularization, IEEE Transactions on Image Processing 14 (2005) 1479-1485.

[22] J. C. Bamber and C. Daft, Adaptive filtering for reduction of speckle in ultrasonic pulse-echo images, Ultrasonics 24 (1986) 41-44. 